\title{
Cognitive derivation of generalization stimuli: Separation of components
}

\author{
HANS O. DOERR \\ Department of Psychiatry and Behavioral Sciences, University of Washington \\ Seattle, Washington 98195
}

\begin{abstract}
In an attempt to separate factors in the generalization of semantic conditioning, a design was utilized in which the subjects (15 each in an experimental and control group) were semantically conditioned through cognitive behavior. Exosomatic GSR was conditioned to a visually presented number (CS) with electrical shock as UCS. During extinction, subjects were presented with visual stimuli consisting of one of three different numbers prefixed by arithmetical symbols and were asked to perform arithmetical operations indicated by series of these visual stimuli. In all series, the number that had served as CS was an intermediate result. The control group was presented the same stimulus slides so rearranged that the CS was never an intermediate result. The GSR of the experimental group during extinction to stimulus slides allowing the derivation of the CS was significantly greater than GSR during any other condition. The findings suggest that cognitive meaning and noteworthiness of the stimulus are critical factors in the generalization of semantic conditioning.
\end{abstract}

The view that thinking is an antecedent rather than a consequence of much of human classical conditioning has gained strong support in recent years (Maltzman, 1971, 1977). There has been a related surge of research into cognitive variables associated with conditioning, such as novelty, meaning, and awareness (Berlyne, 1960; Maltzman, 1977). Among the many problems in the field, those of separating the components of the stimulus variables are prominent (Feather, 1965; Van Olst \& Orlebeke, 1965).

The present study addressed some of these problems within the framework of a semantic conditioning experiment. It received its impetus from Russian work on the interaction of Pavlov's "first and second signal" systems (Fadeyeva, 1959; Sinkovskaia, 1958; Volkova, 1953), in which spoken words served as CSs and visually or verbally presented stimuli served as generalization stimuli. The objective was to study stimulus generalization in a setting in which (1) external or perceptual aspects of stimuli remained the same in both test and control trials and (2) the generalization stimuli had to be derived cognitively by the subjects themselves.

\section{METHOD}

\section{Subjects}

Thirty male undergraduate volunteer subjects were randomly assigned to an experimental or control group. A group size of 15 was the only limitation on randomness.

This research was supported in part by Public Health Service Research Grant MH 06022-05 and in part by Public Health Service Research Training Grant MH 8394-03. Requests for reprints should be sent to Hans O. Doerr, Department of Psychiatry and Behavioral Sciences RP-10, University of Washington, Seattle, Washington 98195.

\section{Apparatus}

Series of stimulus slides were presented with a Carousel projector equipped with a shutter solenoid. Programming units allowed a totally automatic presentation.

Electrical shock was delivered through 16-mm-diam electrodes attached to the ring finger of the left hand. The stimulator was a Grass Model S4 with a SIU 4B stimulus isolation unit. Shock delivery was also programmed.

Respiration and exosomatic GSR were monitored throughout the experiment.

Respiration was monitored through a photocrystal respirometer consisting of a photocrystal and a source of light placed in a sliding tube. The resistance of the photocrystal was modulated as a function of the distance between light and photocrystal. The varying voltage output was written out on a Grass oscillograph.

Exosomatic GSR. A curved $\mathrm{Ag}-\mathrm{AgCl}$ electrode, in contact with a $2-\mathrm{cm}^{2}$ area of skin on the middle finger of the right hand, served as active electrode. A Biophysical Instruments Model 201 GSR amplifier impressed a constant current of $20 \mathrm{~mA}$ at the active site, resulting in a current density of $10 \mathrm{~mA} / \mathrm{cm}^{2}$. A curved silver plate of $58 \mathrm{~cm}^{2}$ area placed on the upper right arm served as the indifferent electrode of the monopolar placement. A .05-molar $\mathrm{NaCl}$ solution suspended in an inert plastic medium (natrosolhydroxyethyl cellulose and polyvinylpyrolidone K-90) was used as electrode paste.

\section{Procedure}

The subjects were placed in a sound-attenuated shielded chamber and were told that the meaning of the various recording devices to be attached would be explained at the termination of the experiment. Following application of the electrodes, a subjectively painful shock threshold was established. Subjects were asked to report when they first felt shock and again when the shock felt distinctly painful. They were then informed that this was the highest level of shock they could expect during the experiment. No information was given as to frequency or time of shock application. Subjects were asked to move as little as possible, not to talk at any time, and to await further instructions to be projected on a screen.

The adaptation period was identical for all subjects. Eight series of slides, containing 10 slides each, were projected. Each 
slide displayed one of three numbers, 2,3 , or 4 , prefixed by one of three arithmetical symbols,,+- , or $X$. The slides were in different order in each series. Common to all eight series was that an intermediate sum, difference, or product was 16, obtained by the subject by following the designated arithmetical procedures. The serial position of the slide at which the subject could derive 16 and the operations by which the subject derived it (addition, subtraction, or multiplication) were counterbalanced in the eight series. The first series of slides was introduced by an instruction slide asking the subject to do the arithmetical operations as indicated. The problems were to be worked out silently; no talking was allowed. Then the problem slides were shown for $6 \mathrm{sec}$ each, at 1 -sec intervals. At the end of each series, an instruction slide informed the subject that one series was completed and that soon the first slide of a new series would be shown.

Following this adaptation period, the acquisition period then followed, and it was also identical for experimental and control subjects. The period was introduced by an instruction slide asking the subject not to perform mental arithmetic during the next slides projected, but just to observe the slides. One series composed of 10 slides was presented. Each slide displayed the number "16." The slides were shown for $10 \mathrm{sec}$ each, at 1 -sec intervals. All subjects were shocked at the established shock level during projection of 6 of the 10 slides, with the shock administered during the last $2 \mathrm{sec}$ of projection. The reinforcement was in an irregular sequence, during projection of Slides 1 , $2,4,6,7$, and 10 . The partial reinforcement schedule was chosen to increase resistance to extinction.

The extinction period followed immediately, preceded by the same instruction slide that had introduced the adaptation period. The subjects were again asked to do the mental arithmetic on the slides to follow. Stimuli and procedures for the experimental group were as during adaptation. The treatment of the control group differed in one significant way: The position of one or two slides in each series was changed in such a way that " 16 " was never an intermediate result.

\section{RESULTS}

\section{Data Reduction}

Responses from each subject during all stimulus periods during adaptation and extinction, for a total of 1606 -sec periods for each subject, were measured for the following variables.

Respiration. Respiration was primarily used as a monitoring variable to observe gross bodily movements, as well as deep breaths, which might initiate changes in the GSR.

Exosomatic GSR. The frequency and magnitude of drops in resistance of $100 \mathrm{ohms}$ or more occurring at an angle of $15 \mathrm{deg}$ or more on the high-gain channel that were not correlated with gross bodily movements or pronounced respiratory changes were recorded. Resistance changes were converted to changes in conductance.

\section{Data Description and Analysis}

The means and standard deviations of the exosomatic GSRs, expressed as changes in skin conductance, are reported in Table 1. The stimulus periods during which a subject could derive "16" (the CS) as an intermediate result are designated as "loaded" periods; all other periods are designated as "nonloaded." Since, during
Table 1

Means and Standard Deviations of Changes in Skin Conductance (Exosomatic GSR) in Micromhos

\begin{tabular}{|c|c|c|c|c|c|c|c|c|}
\hline \multirow[b]{3}{*}{ Treatment } & \multicolumn{4}{|c|}{ Adaptation } & \multicolumn{4}{|c|}{ Extinction } \\
\hline & \multicolumn{2}{|c|}{ L } & \multicolumn{2}{|c|}{ NL } & \multicolumn{2}{|c|}{$\mathrm{L}$} & \multicolumn{2}{|c|}{ NL } \\
\hline & Mean & SD & Mean & SD & Mean & SD & Mean & SD \\
\hline Experimental & .12 & .12 & .14 & .14 & .63 & .54 & .26 & .22 \\
\hline Control & .17 & .21 & .22 & .20 & .30 & .33 & .33 & .30 \\
\hline
\end{tabular}

Note-L = loaded $N L=$ nonloaded.

extinction in the control group, none of the stimulus periods allowed subjects to derive the CS, the periods corresponding in sequence to the loaded periods of the experimental condition were designated as loaded control periods.

Data were analyzed using a "mixed-design" ANOVA, with experimental-control (group) as a between-subjects factor and three within-subjects factors: adaptationextinction (phase), loaded-unloaded (period), and the eight series (series). Significant main effects were obtained for group $[F(1,29)=4.72, p<.05]$, phase $[F(1,930)=$ $11.55, p<.01]$, period $[F(1,930)=7.21, p<.05]$, and series $[F(7,930)=4.40, p<.01]$. A specific hypothesis of the experiment (experimental subjects will show greater GSR responses during the loaded periods in the extinction phase than will controls) was tested by the Group by Phase by Period interaction, and it was supported $[F(1,930)=11.86, p<.01]$. Other significant interactions were Group by Phase $[\mathrm{F}(1,930)=5.58$, $\mathrm{p}<.05]$, Group by Period $[\mathrm{F}(1,930)=7.64, \mathrm{p}<.05]$, Phase by Period $[\mathrm{F}(1,930)=22.65, \mathrm{p}<.01]$, and Phase by Series $[F(7,930)=3.15, p<.01]$.

\section{DISCUSSION}

The results of the present study demonstrate that generalization stimuli can be cognitively derived. The data support Maltzman's (1977) contention that the traditional interpretation of verbal conditioning of the GSR in terms of anxiety, at least generalized anxiety, is invalid. Experimental and control subjects alike were subjected to a noxious situation and found themselves in a situation of uncertainty, conducive to anxiety, in the extinction phase. While there was, indeed, a general increase in the magnitude of skin conductance from the adaptation to the extinction period, the experimental subjects showed a specific increase in response during the loaded periods. Further, stimulus novelty and change could not have played a major role in producing the findings. Both groups had essentially identical external visual stimulus presentations, differing only in slight aspects of sequence. Finally, the contribution of nonspecific, self-generating cortical processes accompanying the subjects' arithmetical problem solving cannot be responsible for the differences observed, since the tasks were matched. What is different between the two groups is the meaning or noteworthiness of the stimuli presented.

In a more general way, the present results imply that selected autonomic responses can be utilized to reflect ongoing cognitive processes in a very sensitive manner. It was already suggested that the technique utilized in this study affords improved control over variables associated with the presentation of conditioned or generalization stimuli. Since the cue stimulus allowing a sub- 
ject to derive the conditioned stimulus is embedded in a series of other stimuli that are highly similar to or identical to the cue stimulus, subjects can be adapted to the physical and novelty aspects of the stimulus presentation. Another use of the method might be in situations requiring the measurement of an intermediate response in a chain of behavior, in which a verbal or motor response could disrupt the ongoing sequence of behavior. Finally, the method might be of use in the study of cognitive behavior in persons who do not have the ability to make verbal or motor responses.

\section{REFERENCES}

Berlyne, D. E. Conflict, arousal and curiosity. New York: McGraw-Hill, 1960.

FADEYEVA, V. K. The peculiarities of the interaction between the first and second signaling systems in the course of formation of conditioned reflexes to complex stimuli in children. In The central nervous system and behavior, selected translations from the Russian medical literature (U.S. Public Health Service Publication No. 762). Washington, D.C: U.S. Government Printing Office, 1959.
Feather, B. Semantic generalization of classically conditioned responses: A review. Psychological Bulletin, 1965, 63, 425-441. Maltzman, I. The orienting reflex and thinking as determiners of conditioning and generalization to words. In H. H. Kendler \& J. T. Spence (Eds.), Essays in neobehaviorism: A memorial volume to Kenneth W. Spence. New York: Appleton-CenturyCrofts, 1971.

Maltzman, I. Orienting in classical conditioning and generalization of the galvanic skin response to words: An overview. Journal of Experimental Psychology, 1977, 106, 111-118.

Sinkovskaia, K. V. The influence of verbal explanation (instruction) on the time taken to reverse salivary and motor conditioned reflexes in children of school age. Pavlov Journal of Higher Nervous Activity, 1958, 8, 611-616.

VAN OLST, E. H., \& OrLebe Ke, J. F. Role of the orienting reflex in the generalization of a conditioned GSR. Canadian Journal of Psychology, 1965, 19, 56-60.

Volkova, V. D. On certain characteristics of the formation of conditioned reflexes to speech stimuli in children. Fiziologicheskii Zhurnal SSSR, 1953, 39, 540-548.

(Received for publication January 7, 1981.) 